DIVISION OF THE HUMANITIES AND SOCIAL SCIENCES

CALIFORNIA INSTITUTE OF TECHNOLOGY

PASADENA, CALIFORNIA 91125

A SOLUTION TO MATCHING WITH PREFERENCES OVER COLLEAGUES

Federico Echenique and Mehmet B. Yenmez

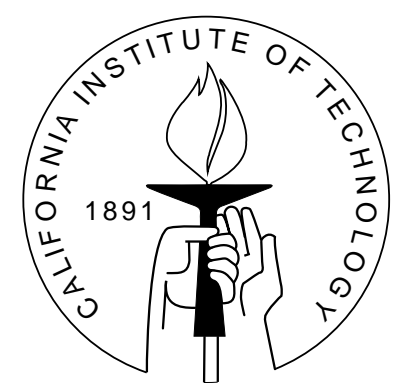

SOCIAL SCIENCE WORKING PAPER 1226 


\title{
A Solution to Matching with Preferences over Colleagues
}

\author{
Federico Echenique and Mehmet B. Yenmez
}

\begin{abstract}
We study many-to-one matchings, such as the assignment of students to colleges, where the students have preferences over the other students who would attend the same college. It is well known that the core of this model may be empty, without strong assumptions on agents' preferences. We introduce a method that finds all core matchings, if any exist. The method requires no assumptions on preferences. Our method also finds certain partial solutions that may be useful when the core is empty.
\end{abstract}

JEL classification numbers: C65,C78

Key words: Matching markets, Core, Lattice, Gale-Shapley Algorithm 


\title{
A Solution to Matching with Preferences over Colleagues *
}

\author{
Federico Echenique and Mehmet B. Yenmez
}

\section{Introduction}

The many-to-one matching model is a commonly-used model of how workers are assigned to firms, or how students are assigned to schools. The model assumes that students do not care who the other students matched to the same school are. This assumption seems problematic for two reasons. First, it is crucial to obtaining the results in the literature: it is widely recognized that the results break down without it. Second, while crucial, the assumption is unlikely to hold in some important applications. Indeed, in many labor markets (such as the academic market) the set of colleagues is an important consideration in choosing whom to work for. In school choice, it seems that students, and their parents, care primordially about colleagues.

In this paper, we study the matching model when students do care about who else goes to the same school. Our approach is not (mainly) to obtain a general structure on preferences that will guarantee existence of some solution to the model. Instead, we propose an algorithm that will find the solutions if they exist.

Our approach is motivated by a certain pessimism. It seems that general conditions for nonemptiness of the core are difficult to obtain, and that the few that are known are very strong. We choose then to be agnostic about the emptiness of the core; we present an algorithm that works without any structure on preferences and that finds the core when it exists. The algorithm also identifies certain partial solutions; these may be useful when the core is empty. In a partial solution that we call "core with singles," the agents who are matched are matched in a stable way, and blocks can only involve agents who are single.

A second motivation is that, in practical problems, where one needs to devise a centralized matching procedure, it is often difficult to verify that agents' preferences

*We thank Chris Chambers, Gary Lorden, and seminar audiences at the University of Arizona, Brown, UCLA, and Princeton, for comments. We are also grateful to Caltech for a SURF fellowship awarded to Yenmez. 
satisfy this or that property. We believe our algorithm will then be useful, as it is guaranteed to work for any preferences.

Our main results hold without any structure on agents' preferences, but we study the behavior of our algorithm under some restrictions on preferences that will ensure a nonempty core. Under these restrictions the algorithm is efficient.

In the rest of the Introduction, we relate this paper to the existing literature.

Nearly all publications on the many-to-one model rule out that a student may care

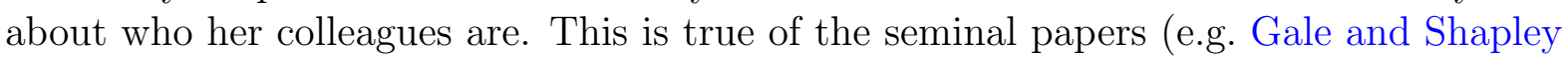

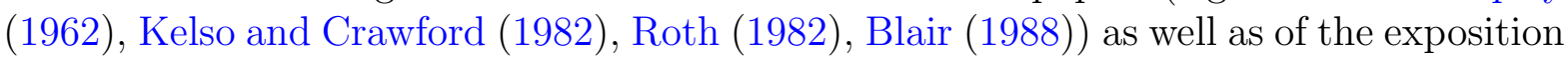

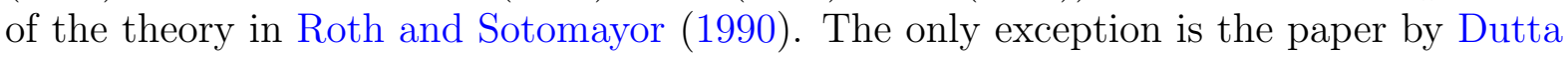

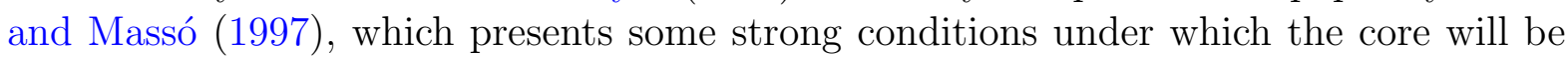
nonempty. Dutta and Massó essentially study lexicographic preferences: the students either first care about the college, then about their colleagues, in which case the core is nonempty; or they care first about their colleagues and then about the college, in which case they need additional assumptions for the core to be nonempty.

Our paper is also related to the literature on matching with couples ( $\bar{R}^{-} \overline{o t h}(19 \overline{8} \overline{4})$,

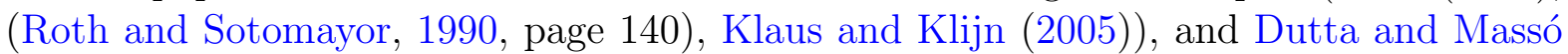
(1997). This literature is mainly motivated by the role of married couples in medical-

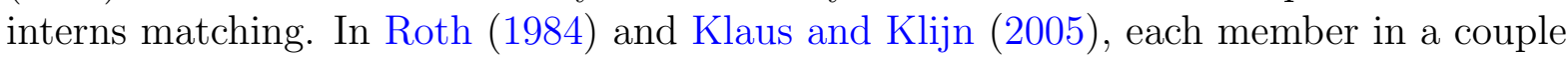
cares about the school choice of his/her partner. But the model is different from ours because couples make a joint decision, and care about the school choice of a partner,

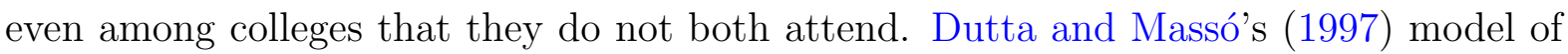
couples is in the spirit of our model of preferences regarding colleagues. In Section $\overline{8}_{1}^{\prime}$ we present an extension of our model to the model with couples in Dutta and Massó; our algorithm can thus be used to find all the core matchings in that model.

For our model of matching with couples, we give a solution to Open Problem 4 in "Roth and Sotomayor" (1990i): when does the pairwise stable set coincide with the core.

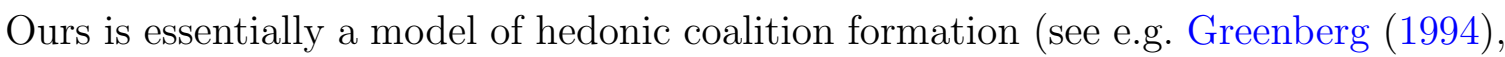

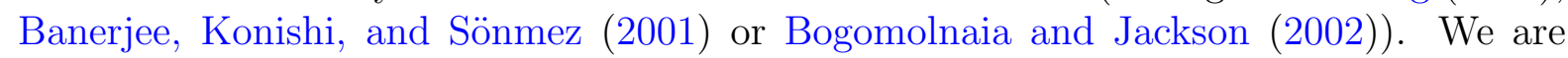
able to adapt some preference restrictions from the coalition-formation literature and use them in our approach. We also note that the method presented here should be easily applicable to the study of stability in more general coalition-formation models.

Finally, we should mention the literature on finding all core matchings- see i $\bar{G} \overline{\text { usfiele }} \overline{\mathrm{d}}$

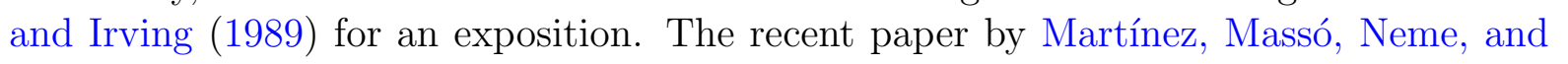
Oviedo $(2004)$ presents an algorithm for the many-to-many case. These papers assume an absence of preferences over colleagues.

We present our model in Section $\overline{2}_{1}^{\prime}$ and give a statement of the problem and outline of our solution. We translate finding the core into a fixed-point problem in Sections $\overline{3}_{-1}^{\prime}$ 
and $\overline{4}_{-}^{\prime}$ In Section $\overline{5}_{-}^{\prime}$ we present the algorithm and discuss partial solutions in Section $\overline{6}_{-}^{\prime}$ In Section $\overline{1}_{-1}^{\prime}$ we restrict preferences to obtain the existence of core matchings. In Section $\overline{8}_{-1}^{\prime}$ we develop a model with couples.

\section{Statement of the Problem}

We state the problem by first specifying a model of matchings with preferences over colleagues and defining the notion of the core. We then outline the difficulties created by preferences over colleagues, and sketch our main contributions.

\subsection{The Model}

There are two disjoint sets of agents, the set of $n$ colleges, $C$, and the set of $m$ students, $S$. Each college $c$ has a strict, transitive, and complete preference $P(c)$ over $2^{S}$. Each student $s$ has a strict, transitive, and complete preference $P(s)$ over $C \times S_{s} \cup\{(\emptyset, \emptyset)\}$; where $S_{s}$ is the set of subsets of $S$ which contain $s$. A preference profile is a collection of preference relations for all colleges and students- that is, an $(n+m)$-tuple $P=$ $\left(P\left(c_{1}\right), \ldots, P\left(c_{n}\right), P\left(s_{1}\right), \ldots, P\left(s_{m}\right)\right)$. A college admissions problem is a 3 -tuple $\langle C, S, P\rangle$.

A matching $\mu$ is a mapping defined on the set $C \cup S$ which satisfies for all $c \in C$ and $s \in S:$

1. $\mu(s) \in C \times S_{s} \cup\{(\emptyset, \emptyset)\}$.

2. $\mu(c) \in 2^{S}$.

3. If $s \in \mu(c)$ then $\mu(s)=(c, \mu(c))$.

4. If $\mu(s)=\left(c, S^{\prime}\right)$ for some college $c$ then $\mu(c)=S^{\prime}$.

Here, $\mu(s)=(\emptyset, \emptyset)$ means that $s$ is not matched to any college. Similarly, if $\mu(c)=\emptyset$ then there are no students matched to college $c$.

Notation. Given a preference relation of a college $c, P(c)$, and a group of students $S^{\prime}$, let $C h\left(S^{\prime}, P(c)\right)$ denote the choice set of $S^{\prime}$ according to $P(c)$; that is, for every $A \subseteq S^{\prime}$ we have $C h\left(S^{\prime}, P(c)\right) R(c) A$. Since $P(c)$ is strict, $C h\left(S^{\prime}, P(c)\right)$ is well-defined.

A matching $\mu$ is individually rational if $\mu(s) R(s)(\emptyset, \emptyset)$ for all students $s$ and $\mu(c)=$ $C h(\mu(c), P(c))$ for all colleges $c$.

A triple $\left\langle C^{\prime}, S^{\prime}, \mu^{\prime}\right\rangle$, where $C^{\prime} \subseteq C, S^{\prime} \subseteq S$ and $\mu^{\prime}$ is a matching, is a block of $\mu$ if the following hold: 
1. $C^{\prime} \cup S^{\prime} \neq \emptyset$; at least one agent is involved.

2. For all $c \in C^{\prime}$ and $s \in S^{\prime}, \mu^{\prime}(c) \in 2^{S^{\prime}}$ and $\mu^{\prime}(s) \in C^{\prime} \times S_{s}^{\prime} \cup\{(\emptyset, \emptyset)\}$; the agents in $C^{\prime} \cup S^{\prime}$ can implement $\mu^{\prime}$ without outside help.

3. For all $f \in C^{\prime} \cup S^{\prime}, \mu^{\prime}(f) R(f) \mu(f)$; all agents in $C^{\prime} \cup S^{\prime}$ are weakly better of.

4. There exists $f \in C^{\prime} \cup S^{\prime}$ such that $\mu^{\prime}(f) P(f) \mu(f)$; at least one agent is strictly better off.

The core is the set of matchings for which there is no block, denoted by $C_{W}(P)$.

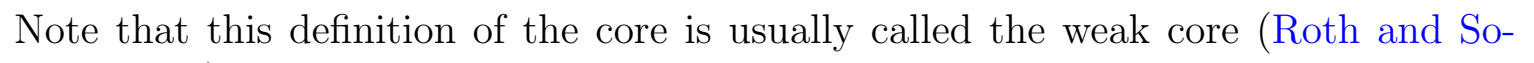
'tomayor, '1990').

\subsection{Problem created by preferences over colleagues.}

It is well-known among specialists in matching that preferences over colleagues creates problems for core existence. We illustrate the problems by an example. The example has an empty core, and nothing obviously pathological - for instance, colleges' preferences satisfy Kelso-Crawford "substitutability." See Section '근. $\overline{3}_{1}^{\prime}$ for a more substantive explanation of the source of problems.

Example 1. Consider two colleges $c_{1}, c_{2}$ and three students $s_{1}, s_{2}, s_{3}$ with the following preferences:

$$
\begin{array}{ll}
P\left(c_{1}\right): & \left\{s_{1}, s_{2}\right\},\left\{s_{1}, s_{3}\right\},\left\{s_{1}\right\},\left\{s_{2}\right\},\left\{s_{3}\right\} \\
P\left(c_{2}\right): & \left\{s_{2}, s_{3}\right\},\left\{s_{3}\right\},\left\{s_{2}\right\} \\
P\left(s_{1}\right): & \left(c_{1},\left\{s_{1}, s_{2}\right\}\right),\left(c_{1},\left\{s_{1}, s_{3}\right\}\right),\left(c_{1},\left\{s_{1}\right\}\right) \\
P\left(s_{2}\right): & \left(c_{2},\left\{s_{2}, s_{3}\right\}\right),\left(c_{1},\left\{s_{1}, s_{2}\right\}\right),\left(c_{1},\left\{s_{2}\right\}\right),\left(c_{2},\left\{s_{2}\right\}\right) \\
P\left(s_{3}\right): & \left(c_{1},\left\{s_{1}, s_{3}\right\}\right),\left(c_{2},\left\{s_{2}, s_{3}\right\}\right),\left(c_{2},\left\{s_{3}\right\}\right) .
\end{array}
$$

This notation means that $c_{1}$ prefers $\left\{s_{1}, s_{2}\right\}$ to $\left\{s_{1}, s_{3}\right\},\left\{s_{1}, s_{3}\right\}$ to $\left\{s_{1}\right\}$, and so on. The potential groups of students not listed are worse for $c_{1}$ than being single.

It is easy to check that, in an individually-rational matching, every student is matched to a college. There are three such matchings:

$\mu_{1}: \begin{array}{llll}c_{1} & c_{2} \\ s_{1} s_{2} & s_{3}\end{array} \quad \mu_{2}: \begin{array}{lll}c_{1} & c_{2} \\ s_{1} s_{3} & s_{2}\end{array} \quad \mu_{3}: \begin{array}{ll}c_{1} & c_{2} \\ s_{1} & s_{2} s_{3}\end{array}$

Now, $\mu_{1}$ is blocked by $\left\langle\left\{c_{2}\right\},\left\{s_{2}, s_{3}\right\}, \mu_{3}\right\rangle, \mu_{2}$ is blocked by $\left\langle\left\{c_{1}\right\},\left\{s_{1}, s_{2}\right\}, \mu_{1}\right\rangle$, and $\mu_{3}$ is blocked by $\left\langle\left\{c_{1}\right\},\left\{s_{1}, s_{3}\right\}, \mu_{2}\right\rangle$. 


\subsection{Outline of our solution.}

We show that the core matchings coincide with the fixed points of a certain function $T$. Motivated by the discussion above, we do not then impose a structure on preferences that will let us prove the existence of fixed points. Instead, we present an algorithm that finds fixed points of $T^{2}$, the composition of $T$ with itself. The fixed points of $T$, and thus the core matchings, are also fixed points of $T^{2}$. Our algorithm may not find all the fixed points of $T^{2}$, but it will find all the fixed points of $T$ - or report that the core is empty if that is the case. Hence we have an algorithm that finds all the matchings in the core, when it is nonempty.

When the core is empty, our algorithm identifies matchings where a subset of the agents are matched in a stable way - their assignments will not be blocked. Other agents are left single in these matchings, and they may block their assignments.

We present some structure on preferences that will guarantee that the core is nonempty, and that our algorithm will find the core quickly.

\section{The Core as a set of fixed points}

We present a construction that allows us to characterize the core as the fixed points of a certain function. This type of construction has been used in the matching literature

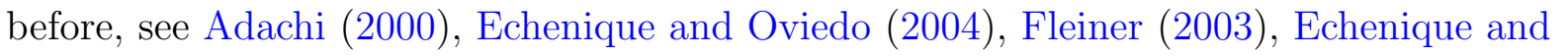
'Oviedo' (2003), 'Hatfield and Milgrom' (2004).

A prematching is a mapping $\nu$, defined on the set $C \cup S$, which satisfies, for all $c \in C$ and $s \in S$,

1. $\nu(s) \in C \times S_{s} \cup\{(\emptyset, \emptyset)\}$.

2. $\nu(c) \in 2^{S}$.

Let $\Phi$ denote the set of prematchings $\nu$.

Remark 1. A prematching $\nu$ is a matching if and only if the following hold: (a) If $s \in \nu(c)$ then $\nu(s)=(c, \nu(c))$. (b) If $\nu(s)=\left(c, S^{\prime}\right)$ then $\nu(c)=S^{\prime}$.

We now proceed to define a function $T: \Phi \rightarrow \Phi$. Let $\nu$ be a prematching. We need the following constructions:

$$
\begin{aligned}
U(c, \nu)= & \left\{S^{\prime} \subseteq S: \forall s \in S^{\prime},\left(c, S^{\prime}\right) R(s) \nu(s)\right\} \\
V(s, \nu)= & \left\{\left(c, S^{\prime}\right) \in C \times S_{s}: \forall s^{\prime} \in S^{\prime} \backslash\{s\}\left(c, S^{\prime}\right) R\left(s^{\prime}\right) \nu\left(s^{\prime}\right)\right. \\
& \text { and } \left.S^{\prime} R(c) \nu(c)\right\} \cup\{(\emptyset, \emptyset)\}
\end{aligned}
$$

That is, $U(c, \nu)$ is the collection of sets of students $S^{\prime}$ so that $\left(c, S^{\prime}\right)$ is better than their matches in $\nu$, for each one of them. $V(s, \nu)$ is the set of $\left(c, S^{\prime}\right)$ so that, for each 
student in $S^{\prime} \backslash\{s\}$, and for the college $c$, the matching in which $c$ is matched to $S^{\prime}$ is better than their matches in $\nu$.

Now, define $T: \Phi \rightarrow \Phi$ by $(T \nu)(f)=\max _{P(f)} U(f, \nu)$ if $f \in C$ and $(T \nu)(f)=$ $\max _{P(f)} V(f, \nu)$ if $f \in S$. The function $T$ takes each college to its optimal set of students, out of those who are willing to attend that college as a group, and each student to its optimal college-group of students pair, out of those willing to accept him/her.

Let $\mathcal{E}(T)=\{\nu \in \Phi: \nu=T \nu\}$.

The main result of this section is

Theorem 2. $\mathcal{E}(T)=C_{W}(P)$.

The proof of Theorem $\underline{2}_{1}^{\prime}$ is in Section ${ }^{2} \overline{3} \cdot \overline{2}^{\prime}$

\subsection{An intermediate notion of stability.}

We first introduce a notion of stability that is instrumental in obtaining our results. A pair $(B, c) \in 2^{S} \times C$ blocks $^{*}$ a matching $\mu$ if $B \cap \mu(c)=\emptyset$ and there exists $A \subseteq \mu(c)$ so that for every $s^{\prime} \in A \cup B,(c, A \cup B) P\left(s^{\prime}\right) \mu\left(s^{\prime}\right)$ and $A \cup B P(c) \mu(c)$. A matching is stable* if it is individually rational and there does not exist student-group-college pair that blocks* $\mu$. Denote the set of stable* matchings by $S^{*}(P)$.

Lemma 3. $S^{*}(P)=C_{W}(P)$.

The proof of Lemma $\overline{13}_{-1}^{\prime}$ is in the appendix.

We isolate part of the proof of Theorem $\overline{2}_{1}^{1}$ as Lemma $\overline{1}_{1}^{\prime}$ as it will be useful in other results.

Lemma 4. Let $\mu$ be a matching and $\nu=T \mu$.

1. If $\nu(c) \neq \mu(c)$ then $(c, \nu(c))$ blocks $^{*} \mu$. If $\nu(s) \neq \mu(s)$ then $\nu(s)$ blocks* $\mu$.

2. If $\nu(c)=\mu(c)$ then there is no block* $(c, D)$ of $\mu$, for any $D \subseteq S$. If $\nu(s)=\mu(s)$ then there is no block ${ }^{*}\left(c^{\prime}, D\right)$ of $\mu$, for any $c^{\prime} \in C$ and $D \subseteq S$ with $D \ni s$.

Proof. We first prove ( $(\overline{1})$. Let $\nu(c) \neq \mu(c)$. That $\mu$ is a matching implies $\mu(c) \in U(c, \mu)$; so $\nu(c) P(c) \mu(c)$. That $\nu(c) \in U(c, \mu)$ implies $(\forall s \in \nu(c))((c, \nu(c)) R(s) \mu(s))$. But $\mu$ is a matching, so $\nu(c) \neq \mu(c)$ implies that $(\forall s \in \nu(c))((c, \nu(c)) \neq \mu(s))$. Hence $(\forall s \in$ $\nu(c))((c, \nu(c)) P(s) \mu(s))$

The proof that, if $\nu(s) \neq \mu(s)$, then $\nu(s)$ blocks* $\mu$, is analogous.

We now prove $\left(\overline{2}^{\prime}\right)$. Let $c \in C$ with $\nu(c)=\mu(c)$. Let $D \subseteq S$ be such that $(\forall s \in$ $D)((c, D) R(s) \mu(s))$, then $D \in U(c, \mu)$. But $\mu(c)=\nu(c)$ implies that $\mu(c) R(c) D$. So $(c, D)$ is not a block* of $\mu$. Now let $s \in S$ with $\nu(s)=\mu(s)$. If $\left(c^{\prime}, D\right)$, with $s \in D$ is such that $D R\left(c^{\prime}\right) \mu\left(c^{\prime}\right)$ and $\left(\forall s^{\prime} \in D \backslash\{s\}\right)\left((c, D) R\left(s^{\prime}\right) \mu\left(s^{\prime}\right)\right)$, then $\left(c^{\prime}, D\right) \in V(s, \mu)$. But $\mu(s)=\nu(s)$ then gives $\mu(s) R(s)\left(c^{\prime}, D\right)$, so $\left(c^{\prime}, D\right)$ is not a block* of $\mu$. 


\subsection{Proof of Theorem ${ }^{\prime} 2$ !}

By Lemma ${ }^{\prime} \overline{3}^{\prime}$, it is enough to prove that $S^{*}(P)=\mathcal{E}(T)$.

We need to show that for every $\nu \in \mathcal{E}(T), \nu$ is a matching and that it is stable* and also if $\mu$ is a stable* matching then $\mu$ is a fixed point of $T$.

Now suppose that $\nu \in \mathcal{E}(T)$. We first show that it is a matching.

Since we already know that $\nu$ is a prematching we only need to show the following: (a)If $s \in \nu(c)$ then $\nu(s)=(c, \nu(c))$. (b)If $\nu(s)=\left(c, S^{\prime}\right)$ then $\nu(c)=S^{\prime}$ by Remark '⿳亠口冋.',

(a) $s \in \nu(c)=(T \nu)(c)=\max _{P(c)}\{U(c, \nu)\}$. Therefore, $(c, \nu(c)) R(\hat{s}) \nu(\hat{s})$ for all $\hat{s} \in \nu(c)$ and in particular

$$
(c, \nu(c)) R(s) \nu(s) \text {. }
$$

Thus, we have $(c, \nu(c)) \in V(s, \nu)$. But now $\nu(s)=(T \nu)(s)=\max _{P(s)}\{V(s, \nu)\}$. Therefore,

$$
\nu(s) R(s)(c, \nu(c)) \text {. }
$$

Since $P(s)$ is strict ( $(\overline{1})$, ) and ( $(\overline{2})$ imply that $\nu(s)=(c, \nu(c))$.

(b) $\nu(s)=\left(c, S^{\prime}\right)$. Now, $\nu(s)=(T \nu)(s)=\max _{P(s)}\{V(s, \nu)\}$. Thus, $\nu(s) \in V(s, \nu)$. Therefore, we have

$$
S^{\prime} R(c) \nu(c)
$$

and also that for all $s^{\prime} \in S^{\prime}-\{s\},\left(c, S^{\prime}\right) R\left(s^{\prime}\right) \nu\left(s^{\prime}\right)$. This, along with $\nu(s)=\left(c, S^{\prime}\right)$ implies that $S^{\prime} \in U(c, \nu)$. But $\nu(c)=(T \nu)(c)=\max _{P(c)}\{U(c, \nu)\}$. So we get

$$
\nu(c) R(c) S^{\prime}
$$

Since $P(c)$ is strict $\left(\overline{3}^{\prime}\right)$ and $(\overline{4})$ imply that $\nu(c)=S^{\prime}$.

Now, assume that $(B, c)$ blocks* $^{*}$. Then, there exists $A \in \nu(c)$ such that $(c, A \cup$ $B) P(s) \nu(s)$ for all $s \in A \cup B$ and $A \cup B P(c) \nu(c)$. This implies that $A \cup B \in U(c, \nu)$. Therefore, $\nu(c)=(T \nu)(c)=\max _{P(c)}\{U(c, \nu)\}$ gives us that $\nu(c) R(c) A \cup B$. This is a contradiction to $A \cup B P(c) \nu(c)$.

To finish the proof, we need to show that for every $\mu \in S^{*}(P)$ we have $\mu=T \mu$. This is a direct consequence of Lemma $\overline{4}$, Let $\nu=T \mu$. Since $\mu$ is stable*, there are no blocking coalitions, which implies that $\nu(c)=\mu(c)$ for every college $c$ and $\nu(s)=\mu(s)$ for every student $s$. Thus, $\mu=\nu=T \mu$.

\subsection{Discussion}

The matching literature that uses constructions like the $T$ function usually proceeds by ordering prematchings and then showing that $T$ is monotone increasing. By application of 
Tarski's fixed-point theorem, then, one proves that $\mathcal{E}(T)$, and thus the core, is nonempty. It may be interesting to see where that approach would fail if applied to our model.

The order on prematchings always involves saying that a prematching $\nu^{\prime}$ is larger than another prematching $\nu$ if all agents on one side of the market prefer $\nu^{\prime}$ to $\nu$, while

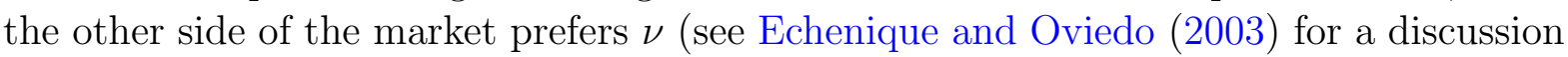
of the two main orders used). Now, if one compares $\bar{T} \nu$ with $\bar{T} \bar{\nu}^{\prime}$ one should get that $T \nu^{\prime}$ is larger than $T \nu$. In the present model, that is a problem because students are choosing their best match out of sets $\left(V(s, \nu)\right.$ and $\left.V\left(s, \nu^{\prime}\right)\right)$ that include agents from both sides of the market. So the set out of which students choose does not depend in a systematic way on the prematching involved. Without preferences over colleagues, since colleges are better off in $\nu^{\prime}$, the set from which students choose shrinks, and thus students prefer $T \nu$ to $T \nu^{\prime}$.

\section{The fixed points of $T^{2}$.}

We have seen that the core can be empty; thus $T$ may not have any fixed points. However, we can prove that $T^{2}$, i.e. the composition of $T$ with itself, must have fixed points. These may not be matchings, let alone core matchings. But if the core is nonempty, the core matchings must be fixed points of $T^{2}$ (and the fixed points of $T^{2}$ that are matchings must be a "partial" solution, see Section ' $\left.\overline{6}^{\prime}\right)$.

The importance of $T^{2}$ becomes clear in Section ${ }^{2} \overline{5}_{\mathrm{p}}^{\prime}$ where we present an algorithm for finding fixed points of $T^{2}$; an algorithm that will find all the fixed points of $T$.

Consider the following partial order on prematchings.

Definition 1. Let $\nu, \nu^{\prime} \in \Phi ; \nu \succ \nu^{\prime}$ if and only if $\nu(f) R(f) \nu^{\prime}(f)$ for all agents $f \in C \cup S$ and $\nu(f) P(f) \nu^{\prime}(f)$ for some agent $f$.

Since the preferences are strict the weak partial order $\succeq$ associated with $\succ$ can be defined as follows: $\nu \succeq \nu^{\prime}$ if and only if $\nu=\nu^{\prime}$ or $\nu \succ \nu^{\prime}$.

Lemma 5. $T$ is monotone decreasing with respect to $\succeq$.

Proof. Let $\nu \succeq \nu^{\prime}$. We are going to show that $T \nu^{\prime} \succeq T \nu$, that is $T \nu^{\prime}(f) \succeq T \nu(f)$ for all agents $f$. We split this into two cases according to whether $f$ is a student or a college:

Let $f \in C$. Let $S^{\prime} \in U(f, \nu)$. Then, for all $s \in S^{\prime},\left(f, S^{\prime}\right) R(s) \nu(s)$. Since $\nu \succeq \nu^{\prime}$, we have $\nu(s) R(s) \nu^{\prime}(s)$. Now, by transitivity we get $\left(f, S^{\prime}\right) R(s) \nu^{\prime}(s)$ for all $s \in S^{\prime}$, which implies that $S^{\prime} \in U\left(f, \nu^{\prime}\right)$. Thus, $U\left(f, \nu^{\prime}\right) \supseteq U(f, \nu)$, which in turn implies

$$
T \nu^{\prime}(f)=\max _{P(f)} U\left(f, \nu^{\prime}\right) R(f) \max _{P(f)} U(f, \nu)=T \nu(f) .
$$

Hence, $T \nu^{\prime}(f) R(f) T \nu(f)$. The proof of $T \nu^{\prime}(f) R(f) T \nu(f)$ when $f \in S$ is analogous. 
Let $\Phi^{\prime}$ be the set of individually-rational prematchings. That is,

$$
\Phi^{\prime}=\{\nu \in \Phi: \forall s \in S, \nu(s) R(s)(\emptyset, \emptyset) \text { and } \forall c \in C, \nu(c) R(c) \emptyset\} \text {. }
$$

When endowed with the partial order $\succeq, \Phi^{\prime}$ is a complete lattice because it is a product set endowed with a product order (Echenique and Oviedo" $\left.{ }^{2} 20 \overline{0} \overline{4}\right)$.

Note that for all $\nu \in \Phi, T \nu \in \Phi^{\prime}$, so we can regard $T$ as mapping $\Phi^{\prime}$ into $\Phi^{\prime}$.

Let $\mathcal{E}\left(T^{2}\right)=\left\{\nu \in \Phi: \nu=T^{2} \nu\right\}$.

Theorem 6. $\mathcal{E}\left(T^{2}\right)$ is a non-empty complete lattice.

Proof. Consider the partial order $\succeq$. We have shown in Lemma 15,1 that $\mathrm{T}$ is monotone decreasing with respect to this partial order. Thus, if $\nu \succeq \nu^{\prime}$ then $T \nu^{\prime} \succeq T \nu$. Now, apply the same lemma to $T \nu^{\prime}$ and $T \nu$ to get $T^{2} \nu \succeq T^{2} \nu^{\prime}$. We have that $T^{2}$ on $\Phi^{\prime}$ is monotone increasing, and also that $\left(\Phi^{\prime}, \succeq\right)$ is a complete lattice. Tarski's fixed point theorem implies that $\left(\mathcal{E}\left(T^{2}\right), \succeq\right)$ is a non-empty complete lattice.

Proposition 7. No two fixed points of $T$ can be ordered by $\succeq$.

Proof. Assume the contrary: There exist $\mu, \mu^{\prime} \in \mathcal{E}(T)$, such that $\mu \succeq \mu^{\prime}$ and $\mu \neq \mu^{\prime}$. Now, by applying Lemma $\overline{5}^{\prime}$, to this inequality we get $T \mu^{\prime} \succeq T \mu$ - that is $\mu^{\prime} \succeq \mu$. Since $\succeq$ is a preorder, we must have $\mu=\mu^{\prime}$, which is a contradiction.

Proposition 8. There exist two prematchings $\bar{\nu}, \underline{\nu} \in \mathcal{E}\left(T^{2}\right)$ such that for all $\nu \in \mathcal{E}(T)$ $\bar{\nu} \succeq \nu \succeq \underline{\nu}$. Moreover, if one of these two prematchings is also a fixed point of $T$, then $T$ has a unique fixed point.

Proof. The existence of $\bar{\nu}$ and $\underline{\nu}$ follows from Theorem $\underline{1}^{\prime}$, as a complete lattice must have a smallest and a largest element.

Now assume that $\bar{\nu}$ is also a fixed point of $T$. If there were another fixed point of $T$, it would also be a fixed point of $T^{2}$. But by the first part we know that $\bar{\nu}$ is better than this fixed point, which contradicts Proposition $\underline{7}^{\prime}$, The case where $\underline{\nu}$ is a fixed point of $T$ is similar.

Remark 2. By monotonicity of $T^{2}$, the iterations of $T^{2}$ starting at the largest prematching in $\Phi^{\prime}$ will eventually reach the prematching $\bar{\nu}$ from Proposition $\overline{8}_{\mathrm{i}}^{\prime}$,

\section{An Algorithm}

We describe an algorithm and prove that it finds all the core matchings. 


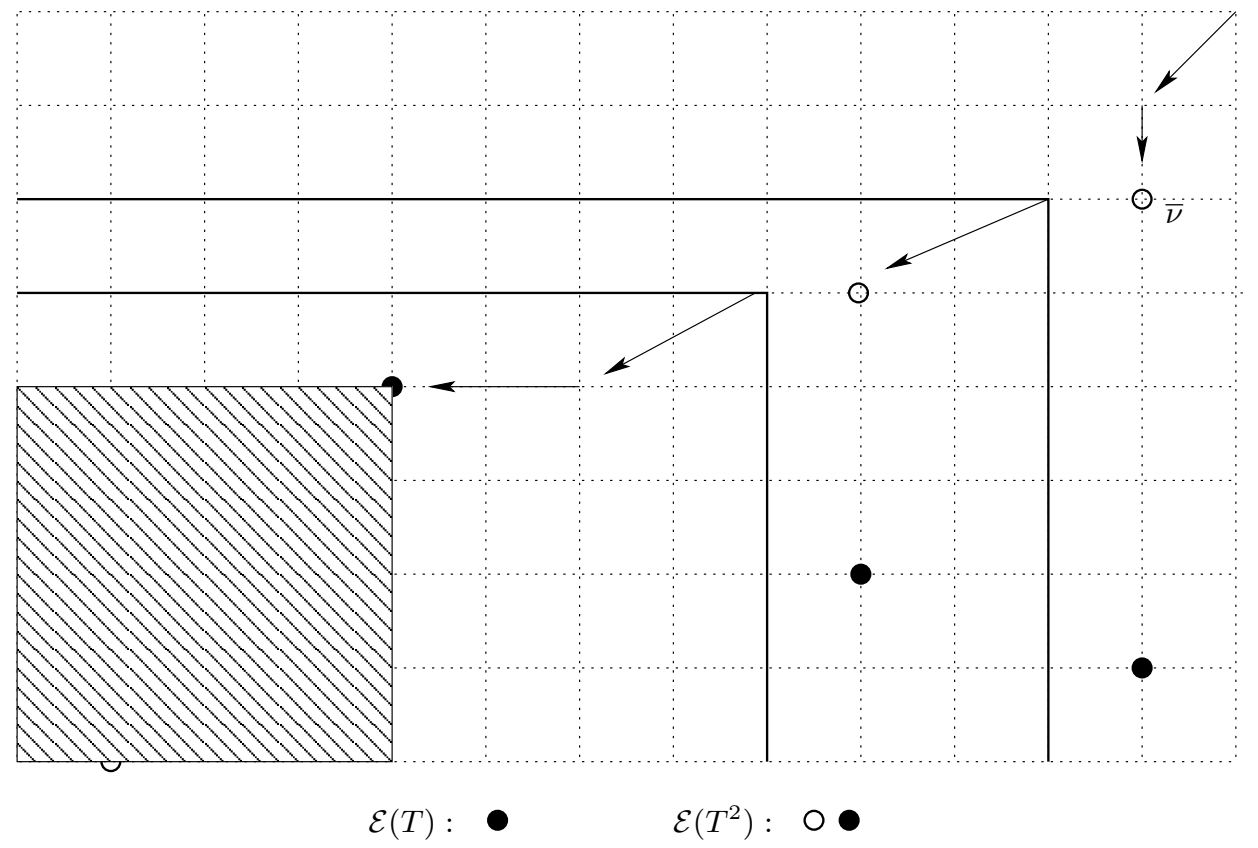

Figure 1: An illustration of the algorithm

\subsection{Description.}

Let $\{1,2, \ldots, n+m\}$ be an enumeration of the elements of $C \cup S$. Given a collegeadmissions problem $\langle C, S, P\rangle$, let $\left\langle F_{1}, F_{2}, \ldots, F_{m+n}\right\rangle$ denote the problem with the same sets of agents, in which each agent's preference list is restricted to those with $F_{f}$ being the top choice for agent $f$. So, in $\left\langle F_{1}, F_{2}, \ldots, F_{m+n}\right\rangle$, agent $f$ finds unacceptable the partners that were originally better than $F_{f}$.

For every agent $f \in C \cup S$ and for every prematching $\nu$, let $i(f, \nu)$ denote the best choice of $f$ that is worse than $\nu(f)$.

Algorithm. Find the smallest $\underline{\nu}$ and largest $\bar{\nu}$ fixed points by applying $T^{2}$ repeatedly to the largest and smallest prematchings in $\Phi^{\prime}$, as suggested in Remark ${ }^{2}{ }_{p}^{2}$ until it finds a fixed point. If $T \bar{\nu}=\bar{\nu}$ then let $\hat{\mathcal{E}}=\{\bar{\nu}\}$ and the algorithm is finished. Otherwise proceed as follows: let $\hat{\mathcal{E}}=\emptyset$. The possible states of the algorithm are the sets of individuallyrational prematchings, and the initial state is $\boldsymbol{Q}=\{\bar{\nu}\}$. While $\boldsymbol{Q} \neq \emptyset$ do the following subroutine.

SUBROUTINE: Set $\boldsymbol{Q}^{\prime}=\emptyset$. For all $\nu \in \boldsymbol{Q}$ and for all $f$ such that $i(f, \nu) R(f) \underline{\nu}(f)$ do steps 1-2 to get a new state $\boldsymbol{Q}^{\prime}$. Then set $\boldsymbol{Q}=\boldsymbol{Q}^{\text {'. }}$

STEP 1. Find the largest fixed point of $T^{2}$ for the problem $\langle\nu(1), \ldots, \nu(f-1), i(f, \nu), \nu(f+1) \ldots, \nu(m+n)\rangle$, call it $\bar{\nu}_{f}$.

STEP 2. If $\bar{\nu}_{f}=T \bar{\nu}_{f}$ then add $\bar{\nu}_{f}$ to $\hat{\mathcal{E}}$; otherwise if $\bar{\nu}_{f} \succ \underline{\nu}$ then add $\bar{\nu}_{f}$ to $\boldsymbol{Q}$ '. 
The algorithm is easy to explain using a picture; see Figure $\overline{1}_{1}^{1}$, The set of prematchings is a product set, and $\succeq$ is a product order. We can represent it as the grid on Figure 1 ; Note how the core matchings, the matchings in $\mathcal{E}(T)$, are unordered, and the matchings in $\mathcal{E}\left(T^{2}\right)$ form a lattice (the smallest element is hidden by the shaded area).

First iterate $T^{2}$ from the largest prematching - represented by the upper right corner. By monotonicity of $T^{2}$ one obtains a monotone decreasing sequence, which has to stop at a fixed point $\bar{\nu}$. Again by monotonicity of $T^{2}, \bar{\nu}$ must be the largest fixed point of $T^{2}$. Now the algorithm iterates $T^{2}$ in a restricted problem, the problem obtained by setting $i(f, v)$ as the best partner for one agent, and $\bar{\nu}(f)$ for everyone else. This restriction is represented by thick lines in Figure ' $1 \bar{L}$, to the left and down of $\bar{\nu}$. By iterating $T^{2}$ we find the largest fixed point in the restricted problem.

The procedure of restricting and finding is repeated. Note that when a core matching is found, we know by Proposition $1 \overline{7}$ t that there cannot be any more core matchings down and to the left. This is illustrated by a shaded area in Figure $\overline{1}_{1}^{\prime}$,

Each restriction changes how $T$ operates, not just the domain of $T$. The successive restrictions can make us lose fixed points of $T^{2}$, but, it turns out, not of $T$ (Proposition $\left.\overline{q_{1}} \bar{p}\right)$. For example, often $\underline{\nu}=T \bar{\nu}$ so that once $\bar{\nu}$ is eliminated, $\underline{\nu}$ is no longer a fixed point of $T^{2}$. The algorithm is based on iEchenique ${ }_{1}^{\prime} \mathrm{s}(20 \overline{0} \overline{3})$ algorithm for non-cooperative games. Echenique's algorithm searches and finds all the fixed points of a monotone function. On the other hand, our algorithm searches for the fixed points of $T^{2}$ but will in general miss some; it is only guaranteed to find all the fixed points of $T$.

\subsection{Results.}

The algorithm proceeds by restricting agents' preferences. Our first result is that one does not lose fixed points with these restrictions.

Proposition 9. If $\mu \in \mathcal{E}(T)$ and $F_{f} R(f) \mu(f)$ for all $f \in C \cup S$, then $\mu$ is also a fixed point of $T$ for the problem $\left\langle F_{1}, F_{2}, \ldots, F_{m+n}\right\rangle$.

Proof. Let $\tilde{T}, \tilde{U}$ and $\tilde{V}$ be the corresponding $T, U$, and $V$ for the restricted problem $\left\langle F_{1}, F_{2}, \ldots, F_{m+n}\right\rangle$. Now, it is clear that $U(c, \mu) \supseteq \tilde{U}(c, \mu)$ for all $c \in C$ and similarly $V(s, \mu) \supseteq \tilde{V}(s, \mu)$ for all $s \in S$. Therefore, $(T \mu)(c) R(c)(\tilde{T} \mu)(c)$ for all $c \in C$ and $(T \mu)(s) R(s)(\tilde{T} \mu)(s)$ for all $s \in S$.

Since $\mu$ is a fixed point of $T$ we have $(T \mu)(c)=\mu(c)$ and $(T \mu)(s)=\mu(s)$.

Now, that $\mu$ is a matching and that $F_{f} R(f) \mu(f)$ for all $f \in C \cup S$ imply $\mu(c) \in \tilde{U}(c, \mu)$ and $\mu(s) \in \tilde{U}(s, \mu)$. Therefore, $(\tilde{T} \mu)(c) R(c) \mu(c)$ and $(\tilde{T} \mu)(s) R(s) \mu(s)$.

To complete the proof we need to put together the inequalities we got: $\mu(c)=$ $(T \mu)(c) R(c)(\tilde{T} \mu)(c) R(c) \mu(c)$ and similarly $\mu(s)=(T \mu)(s) R(s)(\tilde{T} \mu)(s) R(s) \mu(s)$. Since $R(c)$ and $R(s)$ are linear orders we get that $(\tilde{T} \mu)(s)=\mu(s)$ and $(\tilde{T} \mu)(c)=\mu(c)$. 
Theorem 10. $\hat{\mathcal{E}}=\mathcal{E}(T)$, that is, the set $\hat{\mathcal{E}}$ produced by the algorithm above coincides with the fixed points of $T$ which are the core matchings.

Proof. We first show that the algorithm stops at a finite step when $\mathbf{Q}=\emptyset$. Then we establish $\hat{\mathcal{E}} \subseteq \mathcal{E}(T)$ and $\hat{\mathcal{E}} \supseteq \mathcal{E}(T)$ to complete the proof.

Since $\Phi^{\prime}$ is a product set, we can identify $\Phi^{\prime}$ with a grid. Let the distance between each consecutive point in the grid be one unit, and use the resulting Euclidean distance between prematchings. Let $d(\mathbf{Q})$ be the maximum of distances between each prematching in $\mathbf{Q}$ and $\underline{\nu}$ (if $\mathbf{Q}$ is empty let $d(\mathbf{Q})=0$ ). Let $\mathbf{Q}$ and $\mathbf{Q}^{\prime}$ be successive states in the algorithm. It is clear from the definition that $d(\mathbf{Q})>d\left(\mathbf{Q}^{\prime}\right)$. Note that since $\Phi^{\prime}$ is a finite set $d$ takes only a finite number of values. This shows that, after a finite number of steps, we must get $\mathbf{Q}=\emptyset$ which means that the algorithm stops after a finite number of steps.

Now, let us show $\hat{\mathcal{E}} \subseteq \mathcal{E}(T)$. Let $\mu \in \hat{\mathcal{E}}$. This means that $\mu=T \mu$ by Step 2 . Therefore, $\mu \in \mathcal{E}(T)$ which proves $\hat{\mathcal{E}} \subseteq \mathcal{E}(T)$.

To complete the proof we have to show that $\hat{\mathcal{E}} \supseteq \mathcal{E}(T)$. Let $\mu \in \mathcal{E}(T)$. We prove by induction that at every stage $\mathbf{Q}$ of the algorithm, either $\mu \in \hat{\mathcal{E}}$ or there exists $\nu \in \mathbf{Q}$ such that $\nu \succeq \mu$. The beginning state is $\mathbf{Q}=\{\bar{\nu}\}$ and $\hat{\mathcal{E}}=\emptyset$. By the first statement in Proposition $\overline{8}^{\prime}$, we get $\bar{\nu} \succeq \mu$ thus the initial condition is satisfied. Now, let $\mathbf{Q}$ be an intermediate state, from applying the subroutine on a previous state $Q_{0}$. Let $\hat{\mathcal{E}}$ and $\hat{\mathcal{E}}_{0}$ be the associated sets of fixed points. If $\mu \in \hat{\mathcal{E}}_{0}$ then $\mu \in \hat{\mathcal{E}}$ since $\hat{\mathcal{E}} \supseteq \hat{\mathcal{E}}_{0}$. If not then, by the inductive hypothesis, there exists $\nu \in \mathbf{Q}_{0}$ so that $\nu \succeq \mu$. Now, if $\nu=\mu$ then $\mu$ should have already been in $\hat{\mathcal{E}}_{0}$ since at the previous stage in Step 2 it checks for this. Therefore $\nu \succ \mu$. Which implies, by Proposition $1 \overline{9}_{p}^{\prime}$ that there exists $f$ so that $\mu$ is a fixed point of the restricted problem $\langle\nu(1), \ldots, \nu(f-1), i(f, \nu), \nu(f+1) \ldots, \nu(m+n)\rangle$. Now, let $\nu^{\prime}$ be the greatest fixed point of $T^{2}$ for the restricted problem. By Proposition ${ }^{\prime} 7^{\prime}$, either $\nu^{\prime}=\mu$ or $\nu^{\prime}$ is not a fixed point of $T$. If $\nu^{\prime}=\mu$ then $\mu \in \hat{\mathcal{E}}$, otherwise $\nu^{\prime} \in \mathbf{Q}$ and $\nu^{\prime} \succeq \mu$ completing the induction. Now, we have shown in the previous paragraph that the algorithm ends when $\mathbf{Q}=\emptyset$. Hence, the inductive hypothesis implies that $\mu \in \hat{\mathcal{E}}$.

\section{Partial solutions}

What will the algorithm deliver when the core is empty? It turns out that the algorithm can solve the problem partially. It can identify a subset of agents that are matched in a way that will not be blocked, while the rest of the agents block their assignments. We call this partial solution the core with singles.

Definition 2. A matching $\mu$ is in the core with singles if, for any block* $(c, D)$ of $\mu$, $\mu(c)=\emptyset$ and $\mu(s)=(\emptyset, \emptyset)$ for all $s \in D$.

Theorem 11. Let $\mu$ be a matching. If $\mu \in \mathcal{E}\left(T^{2}\right)$, then $\mu$ is in the core with singles. 
It is worth emphasizing that the algorithm does not confuse the core with singles with the core. It identifies the fixed points of $T$, and reports those as the core. But it also finds fixed points of $T^{2}$ that are not fixed points of $T$, and when those are in addition matchings, by Theorem ${ }_{1}^{1} \overline{1}_{b}^{1}$ they must be in the core with singles.

Proof. Let $\nu=T \mu$. We shall first prove that $\mu(c) \neq \emptyset$ implies that $\mu(c)=\nu(c)$, and that $\mu(s) \neq(\emptyset, \emptyset)$ implies that $\mu(s)=\nu(s)$.

Let $c \in C$ be such that $\mu(c) \neq \emptyset$. Since $\mu=T^{2} \mu$, we know that $\mu(c) \in U(c, \nu)$ so $(c, \mu(c)) R(s) \nu(s)$ for all $s \in \mu(c)$. But that $\mu$ is a matching means that $(c, \mu(c))=\mu(s)$; so we have shown that $\mu(s) R(s) \nu(s)$.

On the other hand, that $\mu$ is a matching implies that $\mu(s) \in V(s, \mu)$. This follows from the definition of $V(s, \mu)$, and that $\mu\left(s^{\prime}\right)=(c, \mu(c))$ for all $s \in \mu(c)$. Now, $\nu(s)=(T \mu)(s)$ and $\mu(s) \in V(s, \mu)$ gives $\nu(s) R(s) \mu(s)$. But we proved that $\mu(s) R(s) \nu(s)$, so $\mu(s)=\nu(s)$ follows because $P(s)$ is strict.

Similarly, $\mu=T^{2} \mu$ implies that $\mu(s) \in V(s, \nu)$ for any $s \in \mu(c)$. By the definition of $V(s, \mu)$, then, $\mu(c) R(c) \nu(c)$. But that $\mu$ is a matching implies that $\mu(c) \in U(c, \mu)$; so $\nu(c)=(T \mu)(c)$ implies $\nu(c) R(c) \mu(c)$. Hence $\nu(c)=\mu(c)$.

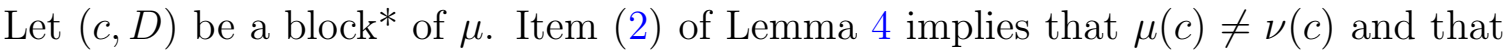
$(\forall s \in D)(\mu(s) \neq \nu(s))$.

Corollary 12. Let $\mu$ be a matching in which no agent is single. Then $\mu$ is a core matching if and only if $\mu \in \mathcal{E}\left(T^{2}\right)$.

Let $\nu$ be a prematching. Denote by $C_{\nu} \subseteq C$ the set of colleges $c$ such that $(c, \nu(c))=$ $\nu(s)$ for all $s \in \nu(c)$. Let $S_{\nu}=\cup_{c \in C_{\nu}} \nu(c)$. Thus the restriction of $\nu$ to $C_{\nu} \cup S_{\nu}$ is a matching.

Proposition 13. Let $\nu \in \mathcal{E}\left(T^{2}\right)$. Then the restriction of $\nu$ to $C_{\nu} \cup S_{\nu}$ is a core matching of $\left\langle C_{\nu}, S_{\nu},\left.P\right|_{C_{\nu} \cup S_{\nu}}\right\rangle$.

Proposition 14. Let $\mu$ be in the core with singles, and let $C^{\prime}$ and $S^{\prime}$ denote the agents who are single in $\mu$. If $\mu^{\prime}$ is in the core with singles of $\left\langle C^{\prime}, S^{\prime},\left.P\right|_{C^{\prime} \cup S^{\prime}}\right\rangle$, then the matching $\left(\mu, \mu^{\prime}\right)$, which matches $C^{\prime}$ and $S^{\prime}$ according to $\mu^{\prime}$, and $C \backslash C^{\prime}$ and $S \backslash S^{\prime}$ according to $\mu$, is in the core with singles of $\langle C, S, P\rangle$.

Proof. Denote the matching $\left(\mu, \mu^{\prime}\right)$ by $\hat{\mu}$. Suppose, by way of contradiction, that there is a block ${ }^{*}\left(c^{*}, S^{*}\right)$ of $\hat{\mu}$ such that the agents in $\left(c^{*}, S^{*}\right)$ are not single under $\hat{\mu}$.

First, suppose that $c^{*} \in C \backslash C^{\prime}$. Then $S^{*} \nsubseteq S \backslash S^{\prime}$, so there is $s \in S^{\prime} \cap S^{*}$. Thus $S^{*} P(c) \mu(c)$, as $\hat{\mu}(c)=\mu(c)$ and $P(c)$ is strict. If we prove that $S^{*} \in U(c, \mu)$ we have reached a contradiction, since $\mu(c)=(T \mu)(c)$ ) (see proof of Theorem refthmcsingles). Now, if $s \in S^{\prime} \cap S^{*}, \mu(s)=(\emptyset, \emptyset)$. Since $\left(c^{*}, S^{*}\right) R(s) \hat{\mu}(s) R(s)(\emptyset, \emptyset)$, we have 
$\left(c^{*}, S^{*}\right) R(s) \mu(s)$. On the other hand, if $s \in S^{*} \backslash S^{\prime}$, then $\hat{\mu}(s)=\mu(s)$ so $\left(c^{*}, S^{*}\right) R(s) \mu(s)$, as $\left(c^{*}, S^{*}\right)$ is a block of $\hat{\mu}$.

Second, suppose that $c^{*} \in C^{\prime}$. Then $S^{*} \nsubseteq S^{\prime}$, as $\left(c^{*}, S^{*}\right)$ cannot be a block of $\mu^{\prime}$. Let $s \in S^{\prime} \cap S^{*}$; then $\left(c^{*}, S^{*}\right) \in V(s, \mu)$. Now we have a contradiction, as before, between $\mu^{\prime}(s)=\left(T \mu^{\prime}\right)(s)$ and $\left(c^{*}, S^{*}\right)$ being a block.

Proposition $1{ }_{1}^{1}{ }_{I}^{\prime}$ suggests a recursive procedure for finding a core matching: run $T^{2}$ to find a matching in the core with singles; put the non-single agents aside; run $T^{2}$ in the reduced market $\left\langle C^{\prime}, S^{\prime},\left.P\right|_{C^{\prime} \cup S^{\prime}}\right\rangle$. This procedure will, in some cases, be very fast.

\section{Restrictions on preferences}

\subsection{The top coalition property}

'Banerjee, Konishi, and $\overline{\text { Sönmez }}\left({ }^{2} \overline{0} 0 \overline{0} \overline{1}\right)$ study coalition-formation games, of which our model is a special case. They introduce the so-called top-coalition property, and prove that it is sufficient for the core to be nonempty and unique. We prove that the topcoalition property is also sufficient for algorithm to find the core efficiently (Theorem, 15 is).

We take the following notational liberty: Let $\mathcal{F}$ be the set of subsets of $C \cup S$ with at most one element from $C$. Let $F=\{c\} \cup \hat{S} \in \mathcal{F}$ and $F^{\prime}=\left\{c^{\prime}\right\} \cup S^{\prime} \in \mathcal{F}$. If $c=c^{\prime}$ we say that $F P(c) F^{\prime}$ if $\hat{S} P(c) S^{\prime}$. If $s \in \hat{S} \cap S^{\prime}$ we say that $F P(s) F^{\prime}$ if $(c, \hat{S}) P(s)\left(c^{\prime}, S^{\prime}\right)$. If $F \subseteq S$, substitute $(\emptyset, \emptyset)$ for $(c, S)$ in the statement above. For all $F \in \mathcal{F}$ with $f \notin F$, say that $\{f\} P(f) F$.

Definition 3. A college-admissions problem satisfies the weak top-coalition property if there exists a partition $\left(F_{1}, F_{2}, \ldots, F_{k}\right)$ of all the agents, where $F_{i} \in \mathcal{F}$ for all $i$, with the following property: For all $f \in F_{1}, F_{1}$ is the top choice in $\mathcal{F}$ for $P(f)$, and for all $f \in F_{i}$, $F_{i}$ is the top choice for $P(f)$ over the sets $F \in \mathcal{F}$ with

$$
F \subseteq(C \cup S) \backslash \cup_{j=1}^{i-1} F_{j}, i=2, \ldots, k .
$$

Theorem 15. If a college-admissions problem satisfies the weak top-coalition property, then it has a unique core matching $\mu$. Moreover, $\mu$ is the largest fixed point of $T^{2}$, and if $k$ is the cardinality of the partition in Definition $13_{p}$ then the algorithm finds $\mu$ in at most $k$ steps.

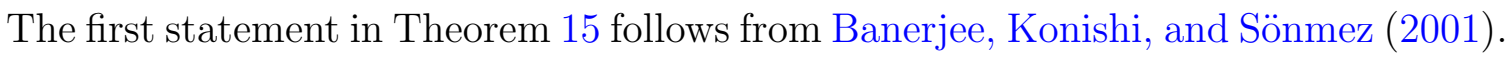
We provide an independent proof to illustrate how our fixed-point method can be used and because we need it to prove the second part of the theorem.

Proof. Let $\left(F_{1}, F_{2}, \ldots, F_{k}\right)$ be a partition of $C \cup S$ which satisfies the weak top-coalition property. Let $\mu$ be the matching which matches every agent in one partition to the agents in that partition. We first prove that $\mu$ is a stable* matching. 
First note that $\mu$ is individually rational since for any agent $f, \mu(f) R(f) \emptyset$ if $f \in C$ or $\mu(f) R(f)(\emptyset, \emptyset)$ if $f \in S$ since $\{f\}$ is also an admissible coalition. Now, $\mu$ is stable* since no agents in $F_{1}$ want to block* since $F_{1}$ is their best choice, no agent in $F_{2}$ wants to block* without the agents in $F_{1}$ since $F_{2}$ is their best choice among $(C \cup S) \backslash F_{1}, \ldots$, no agent in $F_{k}$ wants to block* without the agents in $F_{1} \cup F_{2} \ldots \cup F_{k-1}$.

Uniqueness of $\mu$ follows from Proposition $\overline{8}_{1}^{\prime}$ and by the next part of this theorem that $\mu$ is the largest fixed point of $T^{2}$.

Now, let $\nu_{0}$ be the largest prematching in $\Phi^{\prime}$. Define $\nu_{k}=T \nu_{k-1}$ inductively. Now, $\nu_{0}$ matches each agent in $F_{1}$ to $F_{1}$ since $F_{1}$ is their best choice. $\nu_{1}$ might not match each agent in $F_{2}$ to $F_{2}$ since $F_{2}$ might not be their best overall choice but it still keeps agents in $F_{1}$ matched to $F_{1}$. However, $\nu_{2}$ does match each agent in $f \in F_{2}$ to $F_{2}$ since each agent appearing in $\nu_{1}(f)$ is also an element of $C \cup S-F_{1}$ and $F_{2}=C h\left(C \cup S-F_{1}, P(f)\right)$. It is easy to see with an inductive argument that $\nu_{2(i-1)}$ matches each agent in $F_{1} \cup F_{2} \ldots \cup F_{i}$ to its corresponding coalition for $i=1,2, \ldots, k$. Thus $\nu_{2(k-1)}=\mu$. Since $\mu$ is a stable* matching $\nu_{2 k-1}=T \mu=\mu=\nu_{2(k-1)}$. Thus, we'll be able to get $\mu$ in at most in $2 k-1$ iterations using $T$ or equivalently in at most $k$ iterations using $T^{2}$.

Example ${ }^{1}{ }^{\prime}{ }_{1}^{\prime}$ shows that the weak top-coalition property is not necessary for the result in Theorem $\underline{\underline{1}} \underline{\underline{\underline{\underline{s}}}}$ !

Example 16. Let $S=\left\{s_{1}, s_{2}\right\}$ and $C=\left\{c_{1}, c_{2}\right\}$. Suppose that agents' preferences are:

$$
\begin{array}{ll}
P\left(c_{1}\right): & S,\left\{s_{1}\right\} \\
P\left(c_{2}\right): & S,\left\{s_{2}\right\} \\
P\left(s_{1}\right): & \left(c_{1}, S\right),\left(c_{2}, S\right),\left(c_{2},\left\{s_{1}\right\}\right) \\
P\left(s_{2}\right): & \left(c_{2}, S\right),\left(c_{2},\left\{s_{2}\right\}\right) .
\end{array}
$$

The following array shows the iterations of the algorithm.

\begin{tabular}{c|cccc} 
& $s_{1}$ & $s_{2}$ & $c_{1}$ & $c_{2}$ \\
\hline$\nu_{0}$ & $\emptyset$ & $\emptyset$ & $\emptyset$ & $\emptyset$ \\
$V\left(s, \nu_{0}\right) / U\left(c, \nu_{0}\right)$ & $\left(c_{2}, S\right),\left(c_{2},\left\{s_{1}\right\}\right)$ & $\left(c_{2}, S\right),\left(c_{2},\left\{s_{2}\right\}\right)$ & $\left\{s_{1}\right\}$ & $S,\left\{s_{1}\right\},\left\{s_{2}\right\}$ \\
$\nu_{1}=T \nu_{0}$ & $\left(c_{2}, S\right)$ & $\left(c_{2}, S\right)$ & $\left\{s_{1}\right\}$ & $S$ \\
$V\left(s, \nu_{1}\right) / U\left(c, \nu_{1}\right)$ & $\left(c_{2}, S\right)$ & $\left(c_{2}, S\right)$ & $\emptyset$ & $S$ \\
$T^{2} \nu_{0}$ & $\left(c_{2}, S\right)$ & $\left(c_{2}, S\right)$ & $\emptyset$ & $S$
\end{tabular}

By Proposition ${ }^{\prime} \overline{8}_{\mathrm{p}}^{\prime} T^{2} \nu_{0}$ is the unique core matching. The preferences in this example do not satisfy the weak top-coalition property.

\subsection{Respecting preferences}

We introduce a second restriction on preferences. The assumption is that the "projection" of any agent's preferences to either the set of colleges, or the sets of students, be the same. 
Agents can thus only differ in how they trade off different colleges and students. Under this restriction, the problem turns out to have the weak top-coalition property.

Definition 4. A preference profile $P$ is called respecting if there exist a preference relation $P_{S}$ over $2^{S}$ and a preference relation $P_{C}$ over $C \cup \emptyset$ with the following properties:

1. For all $s \in S,(c, \hat{S}) P(s)\left(c, S^{\prime}\right)$ if and only if $\hat{S} P_{S} S^{\prime}$.

2. For all $s \in S,(c, \hat{S}) P(s)\left(c^{\prime}, \hat{S}\right)$ if and only if $c P_{C} c^{\prime}$.

3. For all $c \in C, \hat{S} P(c) S^{\prime}$ if and only if $\hat{S} P_{S} S^{\prime}$.

4. For all $s \in S$, if $\emptyset P_{C} c$ then $(\emptyset, \emptyset) P(s)(c, \hat{S})$ for all $\hat{S} \subseteq S$.

Proposition 17. If $P$ is respecting then it satisfies the weak top-coalition property.

Proof. Let $F_{1}$ be the union of the top college in $P_{C}$ with the top group of students in $P_{S}$. Clearly, every agent in $F_{1}$ prefers $F_{1}$ to any other coalition. Now, let $F_{2}$ be the union of the top college in $P_{C}$ among the remaining colleges with the top group of students in $P_{S}$ among the remaining group of students. Continue similarly until we exhaust all the colleges c such that $c P_{C} \emptyset$ or all the admissible groups of students $S^{\prime}$ such that $S^{\prime} P_{S} \emptyset$. Then let each remaining agent be a coalition on its own. Assume that we have formed $k$ coalitions. It is clear that $\left(F_{1}, F_{2}, \ldots, F_{k}\right)$ satisfies Definition $\overline{3}_{\mathrm{L}}^{\prime}$

In view of Proposition ' $1 \overline{1} \bar{z}_{b}$, respecting preferences is sufficient for a unique core matching, and for the algorithm to find this core matching in relatively few iterations.

\subsection{Monotonicity of $T$.}

Now order $\Phi$ by $\nu^{\prime} \unrhd \nu$ if, for all $c$ and $s, \nu^{\prime}(c) R(c) \nu(c)$ and $\nu(s) R(s) \nu^{\prime}(s)$. This is the order

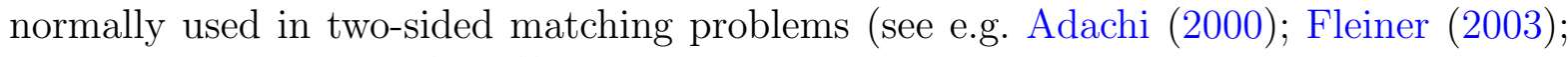
"Echenique and Oviedo" (200 $\overline{4}))$.

The restriction on preferences we shall consider now is that preferences are such that $T$ is monotone increasing, when $\Phi$ is ordered by $\nu^{\prime} \unrhd \nu$.

Proposition 18. If $T$ is monotone increasing, then $\mathcal{E}(T)$ is a non-empty complete lattice. In particular, $\mathcal{E}(T)$ has a smallest (in $\unrhd$ ) element $\underline{\mu}$, and a largest element $\bar{\mu}$. These satisfy, for all $c$ and $s$,

$$
\begin{gathered}
\bar{\mu}(c) R(c) \bar{\nu}(c) R(c) \underline{\nu}(c) R(c) \underline{\mu}(c) \\
\underline{\mu}(s) R(s) \bar{\nu}(s) R(s) \underline{\nu}(s) R(s) \bar{\mu}(s),
\end{gathered}
$$

where $\underline{\nu}$ and $\bar{\nu}$ were defined in Proposition $\overline{1} \overline{\mathrm{s}}$,

Proof. That $\mathcal{E}(T)$ is a non-empty complete lattice follows from Tarski's fixed-point theorem. 
Let

$$
[\underline{\nu}, \bar{\nu}]_{\succeq}=\left\{\nu \in \Phi^{\prime}: \bar{\nu} \succeq \nu \succeq \underline{\nu}\right\}
$$

be the order interval defined by $\underline{\nu}$ and $\bar{\nu}$ using order $\succeq$, and $[\underline{\mu}, \bar{\mu}]_{\unrhd}$ be the corresponding order interval using order $\unrhd$.

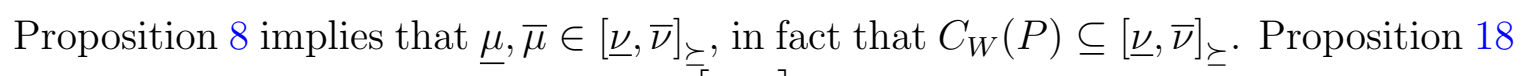
implies that, when $T$ is monotone, $\underline{\nu}, \bar{\nu} \in[\underline{\mu}, \bar{\mu}]_{\unrhd}$

If $T$ is monotone increasing, then, and there is a unique core matching $\mu$, we have $\mu=\bar{\mu}=\mu$. So $\underline{\nu}=\bar{\nu}=\mu$, and our algorithm finds the unique core matching in fewer

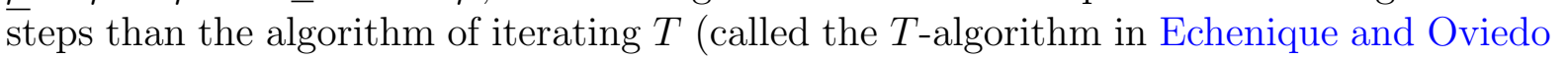
$(\overline{2} \overline{0} \overline{3} \overline{3}))$

\subsection{Preference cycles.}

We show that a type of preference cycle must be present every time a fixed point of $T^{2}$ is not a core matching. So absence of cycles is a useful restriction on preferences. There is nothing pathological about preference cycles, though.

Definition 5. A matching problem $\langle C, S, P\rangle$ exhibits a preference cycle if there is a sequence $\left(\left(c_{1}, S_{1}\right),\left(c_{2}, S_{2}\right), \ldots\left(c_{K}, S_{K}\right)\right)$ such that $\left(c_{1}, S_{1}\right)=\left(c_{K}, S_{K}\right)$ and, for all $k=$ $1, \ldots K-1$, either $c_{k}=c_{k+1}$ and $S_{k+1} P\left(c_{k}\right) S_{k}$ or there is $s \in S_{k} \cap S_{k+1}$ such that $\left(c_{k+1}, S_{k+1}\right) P(s)\left(c_{k}, S_{k}\right)$.

Theorem 19. Let $\mu$ be a matching. If $\mu=T^{2} \mu$ but $\mu \neq T \mu$, then $\langle C, S, P\rangle$ exhibits a preference cycle $\left(\left(c_{1}, S_{1}\right),\left(c_{2}, S_{2}\right), \ldots\left(c_{K}, S_{K}\right)\right)$. Moreover, each $\left(c_{k}, S_{k}\right)$ blocks* $\mu, \mu\left(c_{k}\right)=\emptyset$ for all $k$ and $\mu(s)=(\emptyset, \emptyset)$ for all $s \in \cup_{k} S_{k}$.

Proof. Let $\nu=T \mu$.

STEP 1 Let $\mu(c) \neq \nu(c)$. We shall prove that there is $s \in \nu(c)$ such that $\nu(s) P(s)(c, \nu(c))$,

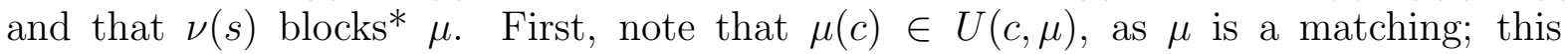
and $\nu=T \mu$ implies that $\nu(c) P(c) \mu(c)$. Now, $\mu(c)=(T \nu)(c)$, since $\mu=T^{2} \mu$. Then $\nu(c) P(c) \mu(c)$ implies $\nu(c) \notin U(c, \nu)$. By definition of $U(c, \nu)$ there must be $s \in \nu(c)$ such that $\nu(s) P(s)(c, \nu(c))$.

Further, $\nu(c) \in U(c, \mu)$ implies that $(c, \nu(c)) R(s) \mu(s)$. So

$$
\nu(s) P(s)(c, \nu(c)) R(s) \mu(s) .
$$

Hence $\nu(s) \neq \mu(s)$. By Lemma ${ }_{L}^{\prime}{ }_{L}^{\prime} \nu(s)$ blocks* $\mu$.

STEP 2 Let $\mu(s) \neq \nu(s)$. We shall prove that there is a block* $\left(c^{\prime}, S^{\prime}\right)$ of $\mu$ such that either (a) $c^{\prime}=\nu(s)$ and $S^{\prime} P(s) \nu(s)$ or (b) there is $\tilde{s} \in \nu(s)$ such that $\nu(\tilde{s}) P(\tilde{s}) \nu(s)$. First, that $\nu(s) \notin V(s, \nu)$ follows analogously to $(\mu(c) \neq \nu(c) \Rightarrow \nu(c) \notin U(c, \nu))$ above. The 
definition of $V(s, \nu)$ implies that either $\nu(\nu(s)) P(\nu(s)) \nu(s)$ or there is $\tilde{s} \in \nu(s)$ such that $\nu(\tilde{s}) P(\tilde{s}) \nu(s)$. Setting $\left(c^{\prime}, S^{\prime}\right)=(\nu(s), \nu(\nu(s)))$ in the first case, and $\left(c^{\prime}, S^{\prime}\right)=\nu(\tilde{s})$ in the second, proves the claim. That $\left(c^{\prime}, S^{\prime}\right)$ is a block* follows applying Lemma ${ }_{L}^{\prime}{ }_{1}^{\prime}$ as in Step 1.

STEP 3 We shall construct a cycle. If there is $c$ with $\mu(c) \neq \nu(c)$, let $\left(c_{1}, S_{1}\right)=$ $(c, \nu(c))$. If there is $s$ with $\mu(s) \neq \nu(s)$, let $\left(c_{1}, S_{1}\right)=\nu(s)$. Let $\left(\left(c_{1}, S_{1}\right),\left(c_{2}, S_{2}\right), \ldots\left(c_{k}, S_{k}\right)\right)$ be a sequence that would be a preference cycle if $\left(c_{1}, S_{1}\right)=\left(c_{k}, S_{k}\right)$, and such that either (a) $\left(c_{k}, S_{k}\right)=\left(c_{k}, \nu\left(c_{k}\right)\right)$ or (b) $\left(c_{k}, S_{k}\right)=\nu(s)$ for some $s \in S_{k}$.

In case (a), by Step 1, there is $s \in \nu\left(c_{k}\right)$ such that $\nu(s) P(s)\left(c_{k}, \nu\left(c_{k}\right)\right)$. Let $\left(c_{k+1}, S_{k+1}\right)=$ $\nu(s)$. Then $\left(c_{k+1}, S_{k+1}\right) \neq \mu(s)$, and $\left(c_{k+1}, S_{k+1}\right)$ blocks* $\mu$. In case (b), by Step 2, either $\nu\left(c_{k}\right) P\left(c_{k}\right) S_{k}$ and $\nu\left(c_{k}\right)$ blocks* $\mu$, or there is $\tilde{s} \in S_{k}$ such that $\nu(\tilde{s}) P(\tilde{s})\left(c_{k}, S_{k}\right)$, and $\nu(\tilde{s}) \neq \mu(\tilde{s})$ is a block* of $\mu$. Let $\left(c_{k+1}, S_{k+1}\right)=\nu\left(c_{k}\right)$, or $\left(c_{k+1}, S_{k+1}\right)=\nu(\tilde{s})$, respectively.

For each element $\left(c_{k}, S_{k}\right)$ in the range of the resulting sequence, $\left(c_{k}, S_{k}\right)$ is in the image of $\nu$. There are finitely many elements in the image of $\nu$, so there is some $K$ such that $\left(c_{1}, S_{1}\right)=\left(c_{K}, S_{K}\right)$.

Corollary 20. Let $\mu$ be a matching and $\mu \in \mathcal{E}\left(T^{2}\right)$. If preferences do not exhibit a preference cycle, then $\mu$ is a stable* matching.

Proof. If $\mu=T \mu$ then $\mu$ is stable* matching by Theorem $\overline{2}^{\prime}$, Otherwise, $\mu \neq T \mu$ which implies that $\langle C, S, P\rangle$ exhibits a preference cycle which is a contradiction.

\subsection{Benchmark: exhaustive search}

The only alternative to our algorithm is to perform an exhaustive search of all possible matchings, and test whether each of them is in the core. We shall argue that our algorithm is more efficient than performing exhaustive search.

The computational task of testing if one matching is in the core is the same as that of computing $T \nu$. To test if a matching is in the core, one needs to see, for each agent, if there is a preferred set of possible partners that would accept the agent. The step of forming the sets $U$ and $V$, and computing the most preferred element, requires the same computation: it can be done as a search from the top down of the agent's preference relation, testing in each case if the potential match would accept the agent.

So the comparison with exhaustive search reduces to comparing how many times the computation of $T \nu$ is done with how many times a match is tested in exhaustive searchthat is, with how many matchings there are in total. For this reason, our algorithm will always do at least as well as exhaustive search. 
Exhaustive search tests all matchings. It is illustrative to calculate how many of these there are, so as to get an idea of the general infeasibility of performing exhaustive search. Pick $k$ of the $n$ colleges to be non-single, this can be done in $\left(\begin{array}{l}n \\ k\end{array}\right)$ ways. Partition the $k$ colleges into $k$ nonempty sets. Each partition then generates $k$ ! different matchings, as there are $k$ ! ways of assigning the elements of the partition to the $k$ colleges. The number of partitions of $m$ elements in $k$ sets is expressed by the Stirling number of the second

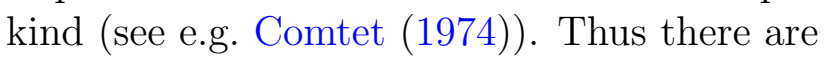

$$
\sum_{k=1}^{n}\left(\begin{array}{l}
n \\
k
\end{array}\right) S_{k}^{m} k !
$$

different matchings. For example, one can assign 1200 students to 9 colleges in $1.233 \times$ $10^{1145}$ different ways.

As we have seen, at least with the top coalition condition our algorithm requires much less computation (each iteration is a computation of $T \nu$ twice). The algorithm may not be quite as efficient under more general preferences, but it probably has an important advantage over exhaustive search, which quickly becomes, for all practical purposes, infeasible.

\section{Extension: A model with Couples.}

We present an extension of our model to a model with couples. The couples introduce a specific form of preferences over colleagues, but it does not reduce to the one we have discussed so far. We present a fixed-point construction, similar to the one above. One can thus use our algorithm to find the core matchings in the model with couples, if there are any. As a by-product, we obtain a result that may be of independent interest: we extend the classical result in the theory of many-to-one matchings, that under KelsoCrawford substitutable preferences the core coincides with a less restrictive pair-wise stable solution. 'L']'

We now assume that there is a subset of students that form couples. So, for each student $s$ in the subset that forms couples, there is one and only one student $s^{\prime}$ so that $s$ forms a couple with $s$ and $s^{\prime}$ forms a couple with $s$.

Split the set of students into two (disjoint) sets, $Q$ and $L$ such that if $s$ forms a couple with $s^{\prime}$ they cannot both be in $Q$ or both in $L$. Thus $Q$ and $L$ form a partition of $S$ that splits all couples. Suppose now that we add a copy of the "singlehood" symbol $\emptyset$ to $Q$ and $L$; in a convenient abuse of notation we shall refer to the different copies by the same label, $\emptyset$. In the sequel, $s$ still denotes a generic element of $S=Q \cup L$, while $q$ and $l$ denote elements of $Q$ and $L$, respectively.

\footnotetext{
${ }^{1}$ This is a solution to Open Problem 4 in "Roth and Sotomayor (1990")
} 
We extend the preferences $P(c)$ to preferences over $2^{Q \times L}$ by: $A P(c) B$, for $A, B \subseteq$ $Q \times L$ if and only if

$$
\begin{array}{r}
\{l: \exists q Q \text { s.t. }(l, q) \in A\} \cup\{q: \exists l \text { s.t. }(l, q) \in A\} P(c)\{l: \exists q \\
\text { s.t. }(l, q) \in B\} \cup\{q: \exists l L \text { s.t. }(l, q) \in B\} .
\end{array}
$$

Note that we abuse notation, using $P(c)$ for the extension of $c$ 's original preferences.

Student $l \in L$ have preferences $P(l)$ over $C \times Q$, and $q \in Q$ has preferences $P(q)$ over $C \times L$.

A prematching is a function $\mu$ on $S \cup C$ such that

1. $\mu(l) \in C \times Q$, if $l \in L$ and $l \neq \emptyset$;

2. $\mu(q) \in C \times L$, if $q \in Q$ and $q \neq \emptyset$;

3. $\mu(c) \subseteq L \times Q$, if $c \in C$.

Let $\mathcal{V}$ be the set of all prematchings.

A matching is a prematching such that, for all $(c, l, q) \in C \times L \times Q$,

$$
\begin{aligned}
& \mu(c) \ni(l, q) \Rightarrow(l \neq \emptyset \Rightarrow \mu(l)=(c, q)) \wedge(q \neq \emptyset \Rightarrow \mu(q)=(c, l)) \\
& \mu(l)=(c, q) \Rightarrow \quad(\mu(c) \ni(l, q)) \wedge(q \neq \emptyset \Rightarrow \mu(q)=(c, l)) \\
& \mu(q)=(c, l) \Rightarrow \quad(\mu(c) \ni(l, q)) \wedge(l \neq \emptyset \Rightarrow \mu(l)=(c, q))
\end{aligned}
$$

\subsection{Stability.}

A matching $\mu$ is individually rational if, for all $c, l, q, \mu(c)=C h(\mu(c), P(c)), \mu(l) P(l)(\emptyset, \emptyset)$ and $\mu(q) P(q)(\emptyset, \emptyset)$.

Let $\mu$ be a matching. A pair $(c,(l, q))$ is a couples-block of $\mu$ if there is some $A \subseteq \mu(c)$ such that

1. $A \cup\{(l, q)\} P(c) \mu(c)$

2. $l \neq \emptyset \Rightarrow(c, q) P(l) \mu(l)$

3. $p \neq \emptyset \Rightarrow(c, l) P(q) \mu(q)$

Note that definition of a couples-block includes the possibility that $(q, \emptyset) \in \mu(c)$ and that $(c,(l, q))$ blocks $\mu$.

A matching is couples-stable if it is individually rational and has no couples blocks. Denote the set of all couples-stable matchings by $S^{c}(P)$. 
A pair $(D, c)$ where $D \subseteq Q \times L$ and $c \in C$ is a block* of a matching $\mu$ if $D R(c) \mu(c)$, for all $(l, q) \in D(c, q) R(l) \mu(l)$ and $(c, l) R(q) \mu(q)$, and if one of the stated relations holds with $P$ in place of $R$.

We state here without proof that the core - denoted $C_{W}(P)$-is the set of matchings for which there is no block*. The proof is very similar to the proof of Lemma $\overline{3}^{3}$,

\subsection{Fixed-point construction.}

To ease notation, when $l=\emptyset$ say that $(c, q) P(l)\left(c^{\prime}, q^{\prime}\right)$ holds by definition. Similarly for $q=\emptyset$.

$$
\begin{array}{r}
V(l, \nu)=\{(c, q):(l, q) \in C h(\nu(c) \cup\{(l, q)\}, P(c))(c, l) R(q) \nu(q)\} \cup\{(\emptyset, \emptyset)\} \\
W(q, \nu)=\{(c, l):(l, q) \in C h(\nu(c) \cup\{(l, q)\}, P(c))(c, q) R(l) \nu(l)\} \cup\{(\emptyset, \emptyset)\} \\
U(c, \nu)=\{(l, q):(c, q) R(l) \nu(l)(c, l) R(q) \nu(q)\} \cup\{(\emptyset, \emptyset)\}
\end{array}
$$

Now let $T: \mathcal{V} \rightarrow \mathcal{V}$ be defined by letting $T \nu(c)=C h(U(c, \nu), P(c))$, and $T \nu(s)$ be the maximal element in $V(s, \nu)$ if $s \in L$ and in $W(q, \nu)$ if $q \in Q$.

Denote the set of fixed points of $T$ by $\mathcal{E}(T)$.

\subsection{Results.}

Lemma 21. If $\mu \in \mathcal{E}(T)$ then $\mu$ is individually rational.

Lemma $2 \overline{1} 1_{1}^{7}$ follows immediately from the definition of the map $T$.

Proposition 22. $\mathcal{E}(T) \subseteq C_{W}(P) \subseteq S^{c}((P)$

Proof. That $C_{W}(P) \subseteq S^{c}(P)$ is immediate. Let $\mu \in \mathcal{E}(T)$ and suppose, by way of contradiction, that $\mu \notin C_{W}(P)$. Let $(D, c)$ be a block* of $\mu$. By definition of a block*, $D \subseteq U(c, \mu)$. So $\mu=T \mu$ implies that $\mu(c) R(c) D$. But $D R(c) \mu(c)$, since $(D, c)$ is a block*.

Now, $D R(c) \mu(c)$ and $\mu(c) R(c) D$ implies that, for all $(l, q) \in D, \mu(l) R(l)(c, q)$ and $\mu(q) R(q)(c, l)$. This is a contradiction with $(D, c)$ being a block*.

Let $c \in C$. Say that $P(c)$ is substitutable if, for any $A, B \subseteq Q \times L$, if $(q, l) \in A \subseteq B$, and $(q, l) \in C h(B, P(c))$ then $(q, l) \in C h(A, P(c))$. Say that a profile $(P(c))_{c \in C}$ is substitutable if each individual $P(c)$ is substitutable. 
Proposition 23. If $(P(c))_{c \in C}$ is substitutable, then $S^{c}(P)=\mathcal{E}(T)$.

Proposition $2 \overline{2} 3_{1}^{3}$ translates into

Corollary 24. If $(P(c))_{c \in C}$ is substitutable, then $S^{c}(P)=C_{W}(P)$.

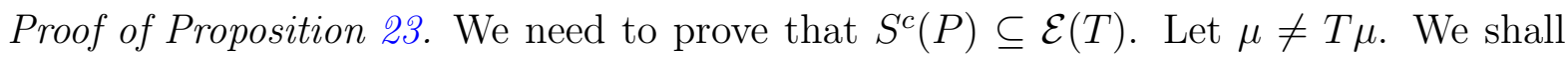
prove that $\mu \notin S^{c}(P)$.

First, suppose there is $c$ such that $T \mu(c) \neq \mu(c)$. Let $D=C h(U(c, \mu), P(c)) \neq \mu(c)$. Since $\mu(c) \subseteq U(c, \mu)$, because $\mu$ is a matching, $D P(c) \mu(c)$. Since $\mu$ is individually rational, $\mu(c)=C h(\mu(c), P(c))$. So we have that $D \nsubseteq \mu(c)$, and hence that there is $(l, q) \in D \backslash \mu(c)$. We shall prove that $(c,(l, q))$ is a couples block of $\mu$. Now,

$$
(l, q) \in C h(U(c, \mu), P(c))=C h(U(c, \mu) \cup\{(l, q)\}, P(c)),
$$

and substitutability of $P(c)$ implies that $(l, q) \in C h(\mu(c) \cup\{(l, q)\}, P(c))$, as $\mu(c) \subseteq$ $U(c, \mu)$. Let $A=C h(\mu(c) \cup\{(l, q)\}, P(c)) \cap \mu(c)$. By definition of $A$, it satisfies (1) in the definition of a couples block.

We now verify (2) and (3) in the definition of a couples block. If $l \neq \emptyset$ then $\mu(l)=$ $\left(c^{\prime}, q^{\prime}\right)$ with $c \neq c^{\prime}$, as $\mu$ is a matching and $(l, q) \notin \mu(c)$. But $(l, q) \in D \subseteq U(c, \mu)$ so $(c, q) R(l) \mu(l)=\left(c^{\prime}, q^{\prime}\right)$. Preferences are strict, so $c \neq c^{\prime}$ implies $(c, q) P(l) \mu(l)$. By the same argument (3) follows.

Second, suppose that there is $l$ such that $T \mu(l) \neq \mu(l)$. Let $(c, q)=T \mu(l)$. We shall prove that $(c,(l, q))$ is a couples block of $\mu$. That $\mu$ is a matching implies $\mu(l) \in V(l, \mu)$. So the definition of $T$ gives $(c, q) P(l) \mu(l)$, requirement (2) in the definition of a couples block.

That $(c, q) \in V(l, \mu)$ implies that

$$
\begin{array}{r}
(l, q) \in C h(\mu(c) \cup\{(l, q)\}, P(c)) \\
(c, l) R(q) \mu(q)
\end{array}
$$

Since $\mu$ is a matching, $(c, q) \neq \mu(l)$ implies $(l, q) \notin \mu(c)$, so Statement $(\overline{5})$ implies $C h(\mu(c) \cup\{(l, q)\}, P(c)) P(c) \mu(c)$. Let $A=C h(\mu(c) \cup\{(l, q)\}, P(c)) \backslash\{(l, q)\} ; A$ satisfies (1) in the definition of a couples block.

Finally, Statement $(\bar{b} \bar{b})$ and that $(c, q) \neq \mu(l)$ implies (3) in the definition of a couples block. 


\section{Appendix: Weaker notions of stability.}

We discuss briefly pairwise stability and $S^{*}(P)$, and prove Lemma ${ }^{\prime}{ }^{\prime}$,in the text. Pairwise stability has been studied widely in many-to-one matchings without preferences over colleagues. The following is the adaptation of pairwise stability to our model:

A pair $(s, c) \in S \times C$ is a pairwise-block of a matching $\mu$ if $s \notin \mu(c)$, but $s \in$ $C h(\{s\} \cup \mu(c), P(c))$ and $\forall s^{\prime} \in C h(\{s\} \cup \mu(c), P(c))$,

$$
(c, C h(\{s\} \cup \mu(c), P(c))) P\left(s^{\prime}\right) \mu\left(s^{\prime}\right) .
$$

A matching $\mu$ is stable if it is individually rational and there does not exist a pairwise block of $\mu$.

Notation. Given a preference profile $P$, we denote the set of stable matchings by $S(P)$.

The following simple proposition and example show that the core is smaller than the set of pairwise stable, and may be strictly smaller. In many-to-one models without preferences over colleagues, the two solutions coincide if colleges' preferences are substitutable (

Proposition 25. $S^{*}(P) \subseteq S(P)$

Proof. Let $\mu \in S^{*}(P)$. Assume that $\mu \notin S(P)$. Since $\mu$ is individually rational there must be a blocking pair $(s, c)$. Hence, $\exists s \in S$ such that $s \notin \mu(c)$, but $s \in C h(\{s\} \cup \mu(c), P(c))$ and $\forall s^{\prime} \in C h(\{s\} \cup \mu(c), P(c)),(c, C h(\{s\} \cup \mu(c), P(c))) P\left(s^{\prime}\right) \mu\left(s^{\prime}\right)$. Therefore, $(\{s\}, c)$ blocks* $\mu$ with $A=C h(\{s\} \cup \mu(c), P(c))-\{s\}$. Contradiction to stability*.

In general, $S^{*}(P)$ can be different from $S(P)$; we make this point through an example. Example 26. Consider three colleges $C=\left\{c_{1}, c_{2}, c_{3}\right\}$ and three students $S=\left\{s_{1}, s_{2}, s_{3}\right\}$ with the following preferences:

$$
\begin{aligned}
P\left(c_{1}\right): & \left\{s_{2}, s_{3}\right\},\left\{s_{2}, s_{1}\right\},\left\{s_{1}, s_{3}\right\},\left\{s_{1}\right\},\left\{s_{2}\right\},\left\{s_{3}\right\} \\
P\left(c_{2}\right): & \left\{s_{2}\right\} \\
P\left(c_{3}\right): & \left\{s_{3}\right\} \\
P\left(s_{1}\right): & \left(c_{1}, s_{1}\right),\left(c_{1},\left\{s_{1}, s_{3}\right\}\right),\left(c_{1},\left\{s_{2}, s_{1}\right\}\right) \\
P\left(s_{2}\right): & \left(c_{1},\left\{s_{2}, s_{3}\right\}\right),\left(c_{2},\left\{s_{2}\right\}\right),\left(c_{1},\left\{s_{2}\right\}\right),\left(c_{1},\left\{s_{1}, s_{2}\right\}\right) \\
P\left(s_{3}\right): & \left(c_{1},\left\{s_{2}, s_{3}\right\}\right),\left(c_{3},\left\{s_{3}\right\}\right) .
\end{aligned}
$$

There is only one stable* matching $\mu_{1}$ which is $\mu_{1}\left(c_{1}\right)=\left\{s_{2} s_{3}\right\}$ and $\mu_{1}\left(c_{2}\right)=\emptyset$ but there is another matching $\mu_{2}$ which is stable and given by $\mu_{2}\left(c_{1}\right)=\left\{s_{1}\right\}, \mu_{2}\left(c_{2}\right)=\left\{s_{2}\right\}$ and $\mu_{2}\left(c_{3}\right)=\left\{s_{3}\right\}$. 
Proof of Lemma 'onst. First we'll show $C_{W}(P) \subseteq S^{*}(P)$ and then $S^{*}(P) \subseteq C_{W}(P)$ to complete the proof.

Let $\mu \in C_{W}(P)$. Since $C^{\prime}=\{c\}, S^{\prime}=\emptyset$ and $C^{\prime}=\emptyset, S^{\prime}=\{s\}$ do not satisfy the definition of a block, for any $\hat{\mu}, \mu$ is individually rational. Moreover, $C^{\prime}=\{c\}$ with any $S^{\prime} \subseteq S$ and $\hat{\mu}$ do not satisfy the definition of a block, so there cannot be any blocking pair involving $c$, so $\mu$ is a stable* matching.

Now we show that $S^{*}(P) \subseteq C_{W}(P)$ by contradiction. Assume that there exists a matching $\mu$ such that $\mu \in S^{*}(P)$ and $\mu \notin C_{W}(P)$. Hence, there exists a coalition $S^{\prime} \cup C^{\prime}$ and $\hat{\mu}$ that satisfy the definition of a block. Therefore, there exists $f \in S^{\prime} \cup C^{\prime}$ so that $\hat{\mu}(f) P(f) \mu(f)$. We split this into two cases:

Case 1. $f \in C$. Then, for all $s \in \hat{\mu}(f), \hat{\mu}(s) R(s) \mu(s)$ by construction of $\hat{\mu}$. Since $\hat{\mu}(f) P(f) \mu(f), \hat{\mu}(f) \neq \mu(f)$ which implies that $\hat{\mu}(s) \neq \mu(s)$ for all $s \in \hat{\mu}(f)$. Since preferences are strict, we get that for all $s \in \hat{\mu}(f), \hat{\mu}(s) P(s) \mu(s)$. Therefore, if we let $B=\hat{\mu}(f)-\mu(f)$ then $(B, f)$ blocks* $\mu$ with $A=\hat{\mu}(f) \cap \mu(f)$, a contradiction to stability* of $\mu$.

Case 2. $f \in S$. Since $\mu$ is a stable* matching, it is individually rational. Thus, $\mu(f) R(f)(\emptyset, \emptyset)$ which implies together with strictness of $P(f)$ and $\hat{\mu}(f) P(f) \mu(f)$ that $\hat{\mu}(f) P(f)(\emptyset, \emptyset)$. Hence, $\hat{\mu}(f)=(c, \hat{\mu}(c))$ for some college $c$. Moreover, $\{c\} \cup \hat{\mu}(c) \in$ $C^{\prime} \cup S^{\prime}$ and $\hat{\mu}$ is at least as good as $\mu$ for all agents in $C^{\prime} \cup S^{\prime}$. Since $\hat{\mu}(f) P(f) \mu(f)$, $\hat{\mu}(f) \neq \mu(f)$. Therefore, $\hat{\mu}(s) \neq \mu(s)$ for all students $s \in \hat{\mu}(c)$ and also $\hat{\mu}(c) \neq \mu(c)$. Now, let $B=\hat{\mu}(c)-\mu(c)$. Hence, the matching $\hat{\mu}$ is better for all students in $\mu(c)$ and also for college $c$. We get that $(B, c)$ blocks* $\mu$ with $A=\hat{\mu}(c) \cap \mu(c)$. A contradiction to stability* $^{*}$ of $\mu$.

\section{References}

AdACHI, H. (2000): "On a Characterization of Stable Matchings," Economic Letters, $68,43-49$.

BanerJee, S., H. Konishi, And T. Sönmez (2001): "Core in a simple coalition formation game," Social Choice and Welfare, 18(1), 135-153.

BlaIR, C. (1988): "The Lattice Structure of the Set of Stable Matchings with Multiple Partners," Mathematics of Operations Research, 13(4), 619-628.

Bogomolnaia, A., and M. O. JaCKson (2002): "The Stability of Hedonic Coalition Structures," Games and Economic Behavior, 38(2), 201-230.

Comtet, L. (1974): Advanced Combinatorics. Kluwer Academic Publishers.

Dutta, B., And J. Massó (1997): "Stability of Matchings When Individuals Have Preferences over Colleagues," Journal of Economic Theory, 75(2), 464-475. 
Echenique, F. (2003): "Finding All Equilibria in Games with Strategic Complements," Caltech SS Working Paper 1153.

Echenique, F., And J. Oviedo (2003): "A Theory of Stability in Many-to-Many Matching Markets," Caltech SS Working Paper 1185.

(2004): "Core Many-to-one Matchings by Fixed Point Methods," Journal of Economic Theory, 115(2), 358-376.

Fleiner, T. (2003): "A Fixed-Point Approach to Stable Matchings and Some Applications," Mathematics of Operations Research, 28(1), 103-126.

Gale, D., And L. S. Shapley (1962): "College Admissions and the Stability of Marriage," The American Mathematical Monthly, 69(1), 9-15.

GreenberG, J. (1994): "Coalition Structures," in Handbook of Game Theory, ed. by R. J. Aumann, and S. Hart, vol. 2, pp. 1305-1337. North Holland.

Gusfield, D., And R. W. Inving (1989): The Stable Marriage Problem: Structure and Algorithms. MIT Press.

Hatfield, J., And P. Milgrom (2004): "Auctions, Matching and the Law of Auctions Matching and the Law of Aggregate Demand," Forthcoming in American Economic Review.

Kelso, A., And V. Crawford (1982): "Job Matching, Coalition Formation, and Gross Substitutes," Econometrica, 50, 1483-1504.

Klaus, B., AND F. KLiJn (2005): "Stable matchings and preferences of couples," Journal of Economic Theory, 121(1), 75-106.

Martínez, R., J. Massó, A. Neme, and J. Oviedo (2004): “An Algorithm to Compute the Full Set of Many-to-Many Stable Matchings," Mathematical Social Sciences, $47(2), 187-210$.

Roth, A., And M. Sotomayor (1990): Two-sided Matching: A Study in GameTheoretic Modelling and Analysis, vol. 18 of Econometric Society Monographs. Cambridge University Press, Cambridge England.

Roth, A. E. (1982): "The Economics of Matching: Stability and Incentives," Mathematics of Operations Research, 7, 617-628.

(1984): "The Evolution of the Labor Market for Medical Interns and Residents: A Case Study in Game Theory," The Journal of Political Economy, 92(6), 991-1016. 